\title{
Bayesian Cluster Characterization and Classification for Direct Numerically Simulated Turbulence Features
}

\author{
Nicholas V. Scott ${ }^{1}$, Jack McCarthy ${ }^{2}$, Robert A. Handler ${ }^{3}$ \\ ${ }^{1}$ Riverside Research, Open Innovation Center, Dayton Research Center \\ 2640 Hibiscus Way, Beavercreek, Ohio, 45431, USA \\ nscott@riversideresearch.org; jack.mccarthy@duke.edu \\ ${ }^{2}$ Duke University, Department of Statistical Science \\ 214 Old Chemistry, Box 90251, Durham, NC, 27708, USA \\ rhandler@gmu.edu \\ ${ }^{3}$ George Mason University, Department of Mechanical Engineering \\ 4400 University Drive, Fairfax, VA, 22030, USA
}

\section{Extended Abstract}

Water shear flow driven by moderate wind stress, an environmental process responsible for the regulation of the exchange of heat and momentum across many spatio-temporal scales, is fundamental to understanding such dynamics as weather and climate change. Central to turbulent shear flow understanding is the use of both laboratory experiments and numerical simulations. Smith et al. [1] acquired infrared imagery measurements of shear driven, surface layer fish-scale thermal instabilities in a laboratory whose structure was substantiated by the structural machine learning analysis of direct numerical simulation (DNS) data of this same scenario performed by Scott and Handler [2]. The efficacy of machine learning analysis in the dimensional reduction of numerically simulated helicity, velocity, and concentration fields suggests that other processing methods could be useful in understanding the turbulent shear flow field structure. Direct numerical simulation data from the work of Scott and Handler [2] was reanalyzed using Bayesian statistical methods in an effort to gain new structural insight into the relationship between these fields.

A 150-point data time series consisting of five dimensions was derived from the simulations and were the result of the random mixing of values from three Reynolds number turbulence regimes of $\operatorname{Re}=150,180$, and 220 . The five dimensions consisted of cross-flow temperature field spatial scale, surface cross-mean flow root mean square (rms) velocity deviation, helicity, rms surface temperature deviation, and Reynolds number. Gaussian mixture modeling [3] of helicity and rms temperature deviation depicts a two-cluster mode covariance structure over high and low rms temperature and helicity levels. Gaussian mixture modeling of helicity and cross-mean flow scales, on the other hand, depicts a two-cluster mode with an isotropic covariance structure suggesting a lack of statistical dependence of the cross-mean flow scales on helicity. This result is conjectured to be due to the particular processing algorithm used in estimation of the cross-mean flow scale width. Hidden Markov model [4] derived emission matrix tables corroborate these trends. Generative topographic mapping using only the first four of the five variables provides mild segmentation compared to the use all five variables, suggesting that low level class distinction is possible when Reynolds number is not used. Bayesian and naïve Bayesian classification [5] was performed for a tri-class distribution where Reynolds number equalling 150,180, and 220 was used as the separation threshold for the causal node. The other four variables were modelled as effect nodes. Assuming equal prior probabilities for each class, naïve Bayesian classification possessed a lower normalized rms deviation than the effect nodal covariance-based Bayesian classification. This suggests that the prediction of Reynolds number from surface scale dynamics is best performed treating helicity, rms temperature and rms cross-mean flow velocity deviation, and cross-mean flow temperature spatial scales independently.

\section{References}

[1] G. Smith, R. A. Handler, and N. Scott "Observations of the structure of the surface temperature field at an air-water interface for stable and unstable cases", in Transport at the Air Sea Interface - Measurements, Models, and Parameterizations, C. S. Garbe, R. A. Handler, and B. Jahne, Eds. Berlin: Springer-Verlag, 2007, pp. 205-222. 
[2] N. V. Scott, and R. A. Handler. (2016, May 17), "Structural characterization of wind-sheared turbulent flow using selforganized mapping", in Proceedings of the Society of Photo-Optical Instrumentation Engineers 9827, Ocean Sensing and Monitoring VIII, 98270X, [Online]. Available: https://doi.org/10.1117/12.2220596

[3] W. L. Martinez, and A. R. Martinez, Computational Statistics Handbook with Matlab. London, UK: Chapman and Hall/CRC, 2001.

[4] L. E. Sucar, Probabilistic Graphical Models. London, UK: Springer, 2015.

[5] S. Theodoridis, A. Pikrakis, K. Koutroumbas, and D. Cavouras, Introduction to Pattern Recognition: A Matlab Approach. Oxford, UK: Academic Press, 2010. 